\title{
Indice desarrollo humano y la tasa de letalidad por Covid-19: Estudio ecológico en América
}

\section{Human development and Covid-19 lethality rate: Ecological study in America}

\section{RESUMEN}

Introducción: El Índice de Desarrollo Humano (IDH), usa tres dimensiones de desarrollo como esperanza de vida, acceso a educación y el nivel de ingresos. Se ha visto a nivel mundial que los grupos socioeconómicamente vulnerables son los menos protegidos y enfrentan el mayor riesgo de COVID-19. Este estudio tiene como objetivo evaluar la correlación entre IDH, índice de inequidad GINI con la tasa de letalidad por COVID-19 en países de América. El estudio: Se desarrolló un estudio observacional ecológico, utilizando las métricas de COVID 19 de países de latino américa. La variable desenlace fue tasa de letalidad por COVID-19, y las variables exposición fueron IDH, GINI, esperanza de vida, años de escolaridad, proporción de mayores de 60 años y PBI per cápita. Recopilado los datos se hicieron análisis. Usamos el método de correlación de spearman, con un nivel de significancia de $95 \%$. Hallazgos: Se evaluaron a 24 países de América Latina, encontrándose una tasa de letalidad por COVID 19 con una mediana de 2.41 y con 1.55 y 3.04 como percentil 25 y 75 respectivamente, obteniéndose además con valores extremos a Ecuador, México y Perú con 6.41, 7.57 y 9.12 respectivamente. Al evaluar la correlación de la tasa de letalidad por COVID 19 se encontró correlación lineal negativa débil entre el IDH, índice de escolaridad y esperanza de vida con $r h o=-0.253,-0.342$ y -0.342 respectivamente, sin embargo no se encontró significancia estadística (Valor $p>0.05$ ). Conclusiones: Se encontró entre los países de latino américa que existe correlación lineal negativa débil entre la tasa de letalidad por COVID 19 y IDH, índice de escolaridad y esperanza de vida.

Palabras Clave: índice de desarrollo humano; letalidad, infección por coronavirus; américa (Fuente: DeCS-BIREME).

\section{ABSTRACT}

Background: The Human Development Index (HDI), uses three dimensions of development such as life expectancy, access to education and income level. It has been seen worldwide that socioeconomically vulnerable groups are the least protected and face the highest risk of COVID-19. This study aims to evaluate the correlation between HDI, GINI inequality index and COVID-19 case
FILIACIÓN

1. Universidad Nacional Mayor de San Marcos, Lima, Perú.

2. Centro de Investigación de Epidemiología Clínica y Medicina Basada en Evidencia (CECLIMBE), Universidad de San Martín de Porres, Lima, Perú.

3. Universidad Continental, Lima, Perú.

4. Universidad San Ignacio de Loyola, Lima, Perú.

5. Universidad Privada Antenor Orrego, Trujillo, Perú.

a. Doctor en Farmacia y Bioquímica.

b. Médico Epidemiólogo.

c. Médico Epidemiólogo y Doctor en investigación Clínica.

\section{ORCID}

1. Mario Carhuapoma-Yance / 0000-0003-4669-6384

2. Moisés Apolaya-Segura / 0000-0001-5650-9998

3. Mario J. Valladares-Garrido / 0000-0003-0839-2419

4. Virgilio E Failoc-Rojas / 0000-0003-2992-9342

5. Cristian Díaz-Vélez / 0000-0003-4593-2509

\section{CORRESPONDENCIA}

Moisés Apolaya-Segura

Dirección: Ca Teodosio Parreño 124 Dpto 604, Barranco, Lima, Perú.

Teléfono: 975391926

EMAIL

moises.apolaya@gmail.com

\section{CONFLICTOS DE INTERÉS}

Los autores niegan conflictos de interés.

FINANCIAMIENTO

Autofinanciamiento.

REVISIÓN DE PARES

Recibido: 20/08/2021

Aceptado: 12/10/2021

\section{COMO CITAR}

Carhuapoma-Yance, M., Apolaya-Segura, M., ValladaresGarrido, M., Failoc-Rojas, V., \& Díaz-Vélez, C. Indice desarrollo humano y la tasa de letalidad por Covid-19: Estudio ecológico en América. Revista Del Cuerpo Médico Hospital Nacional Almanzor Aguinaga Asenjo, 2021, 14(3), 354 - 358. https://doi.org/10.35434/rcmhnaaa.2021.143.1258

Esta obra está bajo una Licencia
Commons Atribución 4.0 Internacional. Commons Atribución 4.0 Internacional. Versión Electrónica: ISSN: 2227-473 Cross Ref. DOI: 10.35434/rcmhnaaa OJS: https://cmhnaaa.org.pe/ojs 
fatality rate in American countries. Study: An ecological observational study was developed, using COVID-19 metrics from Latin American countries. The outcome variable was COVID-19 case fatality rate, and the exposure variables were HDI, GINI, life expectancy, years of schooling, proportion of people over 60 years of age and GDP per capita. Once the data were collected, analyses were performed. We used the spearman correlation method, with a significance level of 95\%. Findings: Twenty-four Latin American countries were evaluated, finding a COVID 19 case fatality rate with a median of 2.41 and with 1.55 and 3.04 as 25th and 75th percentile respectively, obtaining also with extreme values Ecuador, Mexico and Peru with $6.41,7.57$ and 9.12 respectively. When evaluating the correlation of the COVID 19 case fatality rate, a weak negative linear correlation was found between HDI, schooling index and life expectancy with rho $=-0.253,-0.342$ and -0.342 respectively, however, statistical significance was not found ( $p$-value $>0.05$ ). Conclusions: It was found among Latin American countries that there is a weak negative linear correlation between COVID 19 case fatality rate and HDI, schooling index and life expectancy.

Keywords: Human development index; lethality; coronavirus infection; America. (Source: DeCS-BIREME).

\section{INTRODUCCIÓN}

El Índice de Desarrollo Humano (IDH), usa tres dimensiones básicas de desarrollo: esperanza de vida, acceso a educación y el nivel de ingresos. El IDH pretende ser un indicador más confiable de desarrollo en comparación con el crecimiento del Producto Bruto Interno pues considera otras dimensiones además del nivel de ingreso ${ }^{(1)}$.

El IDH se ha propuesto como una posible correlación entre la tasa de infección (proporción de casos confirmados entre la población) y la tasa de letalidad de COVID-19 $9^{(2,3)}$. Un estudio en Brasil, evaluó el Índice de Vulnerabilidad Socioeconómica (SVI) (índice que se basa en características del hogar y el IDH), encontrando que la propagación inicial de COVID-19 se vio afectada principalmente por patrones de vulnerabilidad socioeconómica en lugar de la estructura de edad de la población y la prevalencia de factores de riesgo para la salud ${ }^{(4)}$

En el mundo, los grupos socioeconómicamente vulnerables son los menos protegidos y enfrentan el mayor riesgo de COVID-19, lo que amplía las desigualdades socioeconómicas y de salud inaceptables ${ }^{(5)}$.

La densidad de población es un indicador potencial de la propagación de la infección y está relacionada con las estimaciones de incidencia y mortalidad por COVID-19(6), pero estas estimaciones pueden verse influenciadas por otros factores, incluidos los indicadores socioeconómicos, la adherencia a las políticas de distanciamiento social, la infraestructura de atención de la salud y el grado de conectividad $^{(7)}$.

En este estudio, se pretende evaluar la correlación entre IDH, índice de inequidad GINI, esperanza de vida al nacer, educación, tasa de médicos, proporción de adultos mayores y la tasa de letalidad por COVID-19 en países de américa.

\section{ELESTUDIO}

\section{Población y muestra}

Se desarrolló un estudio observacional ecológico, utilizando las métricas de COVID 19 de países de latino américa publicado en https://www.worldometers.info/coronavirus/ ${ }^{(8)}$, considerando los indicadores de letalidad por cada 100 pacientes por COVID 19; así como los indicadores de los IDH del Programa de las Naciones Unidas para el Desarrollo (UNDP) publicados en http://hdr.undp.org/en/data ${ }^{(9)}$. Se tomaron los datos hasta la fecha del 2 de diciembre del 2021.

\section{Variables}

Se consideraron las siguientes variables para el análisis ${ }^{(8,9)}$ : Tasa de letalidad por COVID 19: Se refiere a la proporción entre el número de pacientes fallecidos por COVID 19 entre el total de pacientes infectados por COVID 19 por 100.

Índice de desarrollo Humano: Un índice compuesto que mide el logro promedio en tres dimensiones básicas del desarrollo humano: una vida larga y saludable, conocimientos y un nivel de vida decente.

Índice de Inequidad (GINI): El coeficiente Gini es el método más utilizado para medir la desigualdad salarial. Es una herramienta analítica que suele emplearse para medir la concentración de ingresos entre los habitantes de una región, en un periodo de tiempo determinado. Medida de la desviación de la distribución del ingreso entre individuos u hogares dentro de un país de una distribución perfectamente equitativa. Un valor de 0 representa igualdad absoluta, un valor de 100 desigualdad absoluta ${ }^{(10)}$.

Índice de Esperanza de Vida: Número de años que podría esperar vivir un recién nacido si los patrones predominantes de tasas de mortalidad específicas por edad en el momento del nacimiento permanecen iguales durante toda la vida del recién nacido.

Índice de Educación: El índice de educación es un promedio de años promedio de escolaridad (de adultos) y años esperados de escolaridad (de niños), ambos expresados como un índice obtenido escalando con los máximos correspondientes

\section{Procedimientos}

Una vez recopilada la información por países, se unieron los datos de las variables a usar en un Microsoft Excel 2010®. Después se procedió a verificar datos erróneos y limpieza de variables.

\section{Análisis de datos}

Para el análisis univariado, las variables cuantitativas se calculó medias y desviación estándar, la mediana, percentil 25 y 75. Para el análisis bivariado se obtuvieron gráficos de 
dispersión y se calculó la correlación entre la tasa de mortalidad por COVID con el índice de desarrollo humano, índice de Inequidad GINI, índice de esperanza de vida y el índice de Educación; Utilizándose el método de correlación de spearman, con un nivel de significancia de $95 \%$. Todos los cálculos fueron realizados con el programa estadístico SPSS versión 24.

Los datos fueron obtenidos de portales web oficiales con bases de datos de acceso abierto, utilizando datos consolidados, sin posibilidad de obtener identificación de los individuos. Por lo cual no requirió aprobación del comité de ética.

\section{HALLAZGOS}

Se registró un total de 24 países, cuyos principales resultados fueron: La mediana de IDH fue 0.77 (0.71 a 0.81), así como la mediana del índice GINI fue 45.1 (41.5 a 48.2). Más detalles se presentan en la tabla 1 . Al evaluar la tasa de letalidad por COVID 19, se puede ver una mediana de 2.86 (1.55 a 3.04), sin embargo se observan valores extremos por encima de 5 en los países como Ecuador, México y Perú con 6.41, 7.57 y 9.12 respectivamente. (Ver figura 1)

Tabla 1. Características generales de los índices evaluados en el presente estudio.

\begin{tabular}{lccccc} 
Características & Media & $\begin{array}{c}\text { Desviación } \\
\text { estándar }\end{array}$ & Mediana & Percentil 25 & Percentil 75 \\
Tasa de Letalidad & 2,86 & $\pm 2,06$ & 2,41 & 1,55 & 3,04 \\
Esperanza de Vida & 75,71 & $\pm 3,69$ & 75,6 & 74,2 & 78,2 \\
Años de Escolaridad & 9,52 & $\pm 2,37$ & 8,95 & 8,3 & 10,75 \\
Índice de Desarrollo Humano & 0,76 & $\pm 0,09$ & 0,77 & 0,71 & 0,81 \\
Médicos por 1000 habitantes & 2,18 & $\pm 1,81$ & 1,75 & 1,2 & 2,6 \\
PBI per cápita & 17276 & \pm 14067 & 13255 & 8524 & 20627 \\
Índice GINI & 44,5 & $\pm 4,54$ & 45,1 & 41,5 & 48,2 \\
\hline
\end{tabular}

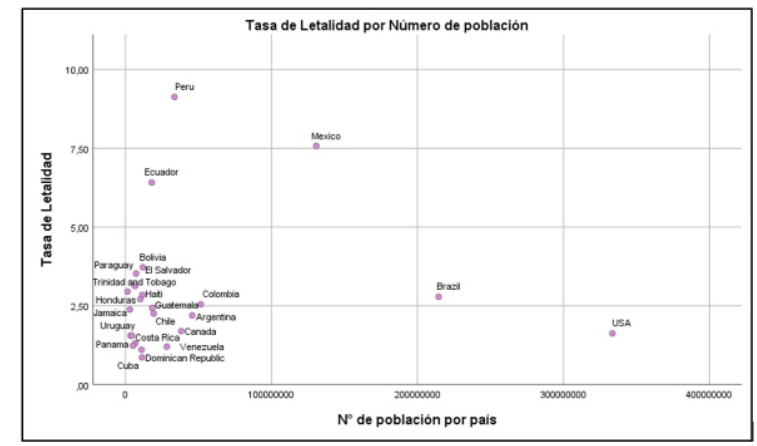

Figura 1.

Tasa de letalidad por COVID 19 según número de población por país latino americano.

En las figuras 2, se muestran los análisis de correlación entre tasa de letalidad por COVID-19 y el IDH, así como el resto de co-variables de interés. Se observó correlación lineal débil negativa entre el IDH y tasa de letalidad por COVID-19 (rho= $0.253 ; p=0.233$ ). Adicionalmente, se evidencia correlación lineal débil negativa entre el Índice de educación (rho= $0,342 ; p=0,102)$ y esperanza de vida ( $r h o=-0,342 ; p=0,102)$.
No se observó correlación con el Índice de inequidad GINI, médicos por cada mil habitantes, PBI per cápita y proporción de adultos mayores de 65 años.
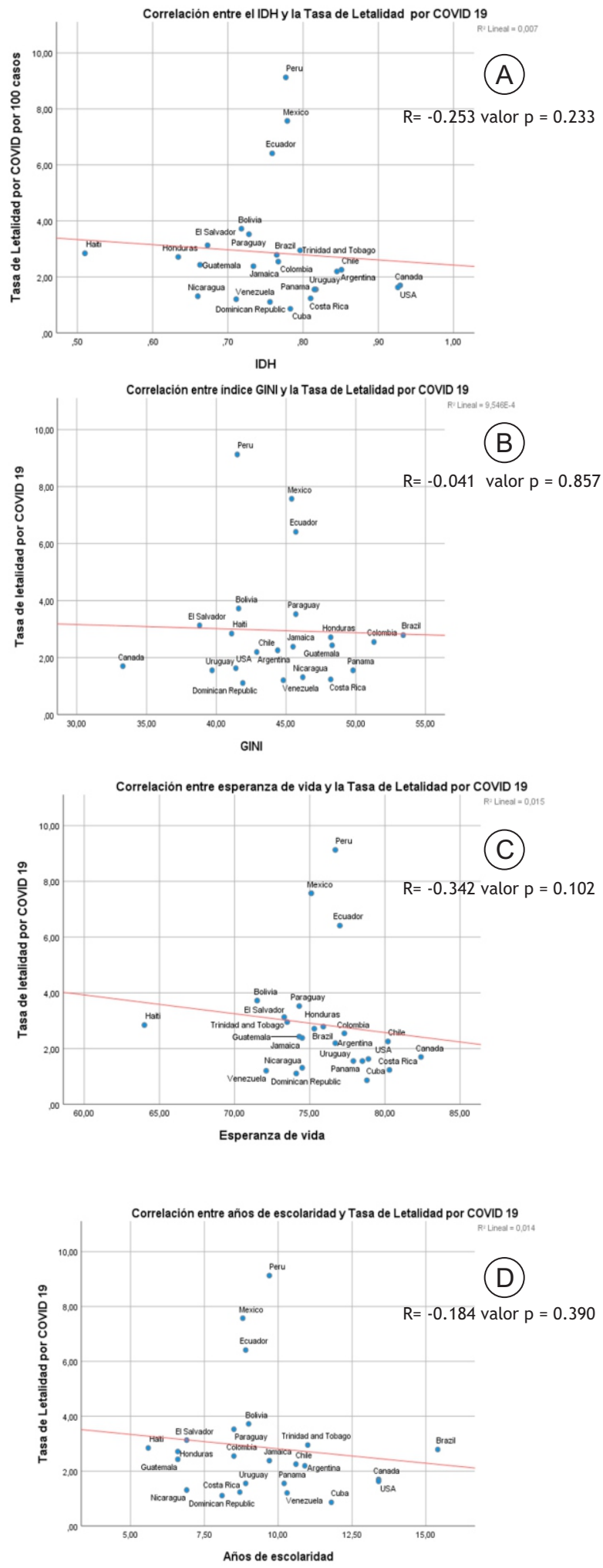

Figura 2.

Correlación del índice de desarrollo humano (IDH), Índice GINI, esperanza de vida, años de escolaridad, proporción mayor de 60 años, PBI per cápita y tasa de letalidad por COVID-19. 

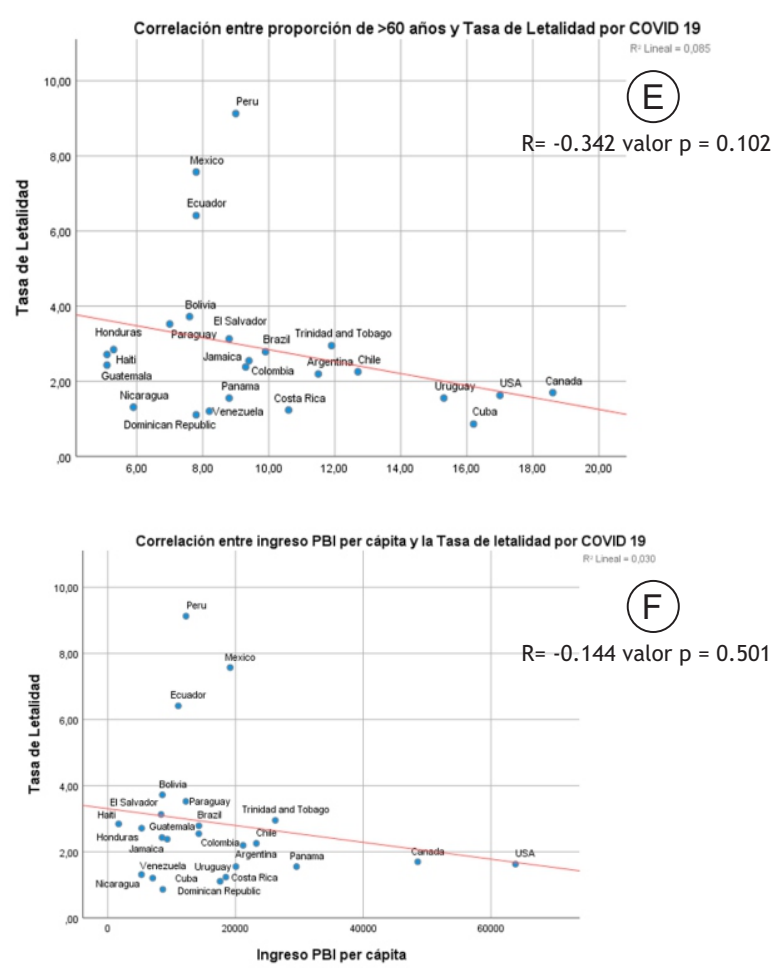

Leyenda: A= Índice de Desarrollo Humano (IDH), B = Indice GINI, C = Esperanza de vida, $\mathrm{D}=$ Años de Escolaridad, $\mathrm{E}=$ Proporción $>60$ años y $\mathrm{F}=\mathrm{PB}$ per Cápita.

Figura 2.

Correlación del índice de desarrollo humano (IDH), Índice GINI, esperanza de vida, años de escolaridad, proporción mayor de 60 años, PBI per cápita y tasa de letalidad por COVID-19.

\section{DISCUSIÓN}

\section{Hallazgos principales}

De los 24 países evaluados, la tasa de letalidad mostró una correlación débil negativa con IDH, índice de escolaridad y esperanza de vida. En todos los análisis se observó que Perú, México y Ecuador mantuvieron datos extremos.

\section{Índices y letalidad por COVID-19}

En este estudio se observó correlación lineal negativa débil entre el IDH y la tasa de letalidad por COVID-19, sin embargo no se evidenció significancia estadística. Esto es distinto de una investigación similar realizada a nivel distrital de Lima Metropolitana, en la cual se observó correlación significativa entre IDH y letalidad por COVID-19 ${ }^{(11)}$, si bien este estudio fue realizado en un solo país, puede que los resultados intraobservaciones (Perú), difiera de lo observado entre grupos (Países de América). Explicado probablemente por ser una evaluación en comunidades distritales más homogéneas en un contexto socioeconómico y cultural similar.

Los hallazgos indican que IDH por sí sólo no genera una asociación favorable sobre la pandemia de COVID 19, lo cual podría explicarse por otras variables que influyen en los sistemas de salud y su contención de la pandemia, así como aspectos de contexto poblacional como aquellos países con mayor esperanza de vida, porcentaje elevado de población mayor de 60 años, ambos puntos condicionan tener una mayor prevalencia de personas con mayores tasas de obesidad y enfermedades pulmonares crónicas tuvieron un riesgo de mortalidad significativamente mayor al inicio de la pandemia con respecto a países donde predominan las enfermedades transmisibles ${ }^{(12)}$. La explicación de esto es un estudio realizado en países del primer mundo, donde encontraron que el hábito de fumar, el porcentaje de población anciana y la frecuencia de las pruebas son las variables más significativas asociadas con la morbilidad y mortalidad $^{(13)}$.

Por otro lado, el índice de educación muestra una correlación inversa débil, aunque no significativa, aporta en sugerir que a mayor nivel de educación de una población fortalece la respuesta ante la recepción de mensajes claves para reducir la posibilidad de contagió a través de medidas de promoción de la salud, y reducir la letalidad al acudir tempranamente a los centros asistenciales. Así la educación, permite tener oportunidades para la prevención y ayuda a orientar a los servicios sanitarios con medidas que atiendan los principales problemas de salud de la comunidad como el contexto COVID y fortalece los servicios de promoción, prevención, tratamiento y rehabilitación necesarios ante diversas coyunturas $^{(14,15)}$.

Esta investigación presenta algunas limitaciones. Primero, al ser un estudio ecológico, no podemos inferir la causalidad de los hallazgos a nivel individual. Segundo, podrían existir problemas en la calidad de información registrada durante la pandemia por COVID-19 en los países evaluados, lo cual conlleva a sesgo de información. Tercero, podría encontrarse potenciales diferencias entre los clusters de los países evaluados, por lo cual, habría que considerarlo para evitar alguna falacia ecológica.

\section{CONCLUSIONES}

Se evidenció correlación lineal negativa débil entre el IDH y la tasa de letalidad por COVID-19, no obstante, no se observó diferencia estadística. Adicionalmente, se observó correlación lineal negativa débil entre tasa de letalidad por COVID-19 y el índice de escolaridad y esperanza de vida.

\section{REFERENCIAS BIBLIOGRÁFICAS}

1. Índice de Desarrollo Humano - IDH [Internet]. Instituto Peruano de Economía. 2011 [citado el 25 de octubre de 2021]. Disponible en: https: / /www.ipe.org.pe/portal/indice-de-desarrollo-humano-idh/

2. Liu K, He M, Zhuang Z, He D, Li H. Unexpected positive correlation between human development index and risk of infections and deaths of COVID-19 in Italy. One Health Amst Neth. diciembre de 2020;10:100174.

3. Varotsos CA, Krapivin VF, Xue Y. Diagnostic model for the society safety under COVID-19 pandemic conditions. Saf Sci [Internet]. el 1 de abril de 2021 [citado el 25 de octubre de 2021];136:105164.
D i s p o n i b l e
e $\mathrm{n}$ : https://www.sciencedirect.com/science/article/pii/S09257535210 00072

4. Rocha R, Atun R, Massuda A, Rache B, Spinola P, Nunes L, et al. Effect of socioeconomic inequalities and vulnerabilities on health-system preparedness and response to COVID-19 in Brazil: a comprehensive analysis. Lancet Glob Health [Internet]. el 1 de junio de 2021 [citado el 25 de octubre de 2021];9(6):e782-92. Disponible en: https: //www.sciencedirect.com/science/article/pii/S2214109X210 00814

5. Ahmed F, Ahmed N, Pissarides C, Stiglitz J. Why inequality could 
spread COVID-19. Lancet Public Health [Internet]. el 1 de mayo de 2020 [citado el 25 de octubre de 2021];5(5):e240. Disponible en: https://www.sciencedirect.com/science/article/pii/\$24682667203 $\underline{00852}$

6. Martins-Fitho PR. Relationship between population density and COVID-19 incidence and mortality estimates: A county-level analysis. $\mathrm{J}$ Infect Public Health [Internet]. agosto de 2021 [citado el 25 de octubre de 2021];14(8):1087-8. Disponible en: https://www.ncbi.nlm.nih.gov/pmc/articles/PMC8253654/

7. Troumbis AY. Testing the socioeconomic determinants of COVID-19 pandemic hypothesis with aggregated Human Development Index. J Epidemiol Community Health. 2021;75(4):414-5.

8. Woldometer. COVID-19 Coronavirus Pandemic [Internet]. Revisado el 02 de noviembre del 2021 . Disponible: https://www.worldometers.info/coronavirus/

9. Human Development Data Center. United Nations Development Programme [Internet]. Revisado el 02 de noviembre del 2021. Disponible: http://hdr.undp.org/en/data

10. Schneider MC, Castillo-Salgado C, Bacallao J, Loyola E, Mujica OJ, Vidaurre $M$, et al. Métodos de medición de las desigualdades de salud. Rev Panam Salud Publica [Internet]. 2002 [citado el 13 de noviembre de 2021 ]; $12: 398-414$. Disponible en: https://scielosp.org/article/rpsp/2002.v12n6/398-414/.

11. Dorregaray-Farge ZE, Soto A, De la Cruz Vargas J. Correlación entre mortalidad por COVID-19, índices de riqueza y desarrollo humano y densidad poblacional en distritos de Lima Metropolitana durante e 2020. Revista de la Facultad de Medicina Humana. 2021: 21(4).doi: 10.25176/RFMH.v21i4.3987.

12. Ferrándiz Espadin Renato, Cieza Zevallos Javier. Relación de los indicadores de desarrollo económico, sociodemográfico, sanitario y social con la tasa de mortalidad por COVID-19 en los primeros 120 días de la pandemia. Rev Med Hered [Internet]. 2021 Jan 32 (1): 20-32. Disponible en: http://dx.doi.org/10.20453/rmh.v32i1.3944.

13. Kumru S, Yiğit P, Hayran O. Demography, inequalities and Global Health Security Index as correlates of COVID-19 morbidity and mortality. Int J Health Plann Manage. 2021 Nov 11:10.1002/hpm.3384. doi: $10.1002 / \mathrm{hpm} .3384$.

14. Guillem FC. Oportunidades y amenazas para la prevención y promoción de la salud y el PAPPS en el contexto de la pandemia Covid19 [Opportunities and threats for prevention and health promotion and the PAPPS in the context of the Covid-19 pandemic]. Aten Primaria. 2020 Aug-Sep;52(7):449-451. Spanish. doi: 10.1016/j.aprim.2020.07.001.

15. Nogueira J, Rocha DG, Akerman M. Políticas públicas adoptadas en la pandemia de la COVID-19 en tres países de América Latina: contribuciones de la Promoción de la Salud para no volver al mundo que existía. Global Health Promotion. 2020. https://doi.org/10.1177/1757975920977837. 